\title{
What an editor wants from your manuscript
}

\section{Editorial}

Published words are of crucial importance in medicine as they may have implications for patients.

The quality and success of a scientific publication is, in addition to the relevance of the topic and the validity of the method used, in accordance with the principles of the scientific medical writing regarding the structure and style. ${ }^{1,2}$ The task of the chief editor of a biomedical journal is not only to read each manuscript sent to the editorial committee before sending it to its reviewers for a peer review.

The editor in chief must also evaluate the content of each manuscript received and give his/her opinion. Although this is an arduous task, at the same time, it is a pleasant activity since as editors, we have the great opportunity to read for the first time a virgin writing that intends to be edited, published and distributed to an audience of medical professionals eager to know new ideas, novel hypotheses, original results, updates, reviews, cases and their potential applications in their patients.

As editor and reviewer of JACCOA, I have been fortunate to read many of the manuscripts that have been received, approved and published in our journal. On the other hand, I have been directly responsible for the rejection of some writings that did not meet the requirements for publication. This is the most difficult part of an editor of a young open access journal, a biomedical journal that has begun recently in the arduous task of transmitting the knowledge and experience of colleagues from around the world who have favored us by sending their manuscripts to our editorial committee.

My experience as a reviewer and editor has taught me that we must have time and be patient with those authors of poor manuscripts. Furthermore, I must say that I love personally guide all authors who are inexperienced writers to encourage them to publish their experiences. One of my first clinical research articles visited three editorial committees before being accepted for publication in an indexed journal. ${ }^{3}$ Recently, and with the experience of more than 130 publications, another of my manuscripts toured 4 editorial committees until it was accepted. ${ }^{4}$ In both manuscripts, I had to make modifications according to the author guides of each of the journals to which they were sent, paying special attention to grammatical expressions, without ignoring the scientific foundations of the contents.

Badly written manuscripts can be fatal, and must be completely corrected. Manuscripts with the potential to be published only require a systematic breakdown of weaknesses, which will improve the quality of the manuscript. Each reviewer should give you a kind, quick and accurate opinion to help you to make proper changes in your original manuscript.

\section{How to make your manuscript accepted}

The quality of an original scientific article depends on compliance with the principles of scientific medical writing in terms of structure and style. ${ }^{1,2,5}$ To avoid your manuscript from traveling from journal to another journal like my examples, you must be cautious when writing your ideas. When structuring your manuscript do it in the
Volume 12 Issue I - 2020

\author{
Victor Whizar-Lugo MD \\ Chief Editor, México
}

Correspondence: Víctor M.Whizar-Lugo MD, Unidad de Cuidados Intensivos, Hospital General de Tijuana, ISESALUD, Tijuana B.C., México, Email vwhizar@anestesia-dolor.org

Received: December 20, 2019 | Published: January 2I, 2020

following order; Introduction, Methods, Results, Discussion and References. Don't forget to write a summary and keywords. Your style must be concise, clear, precise and simple. ${ }^{5,6}$ If English is not your native language, it is recommended that your manuscript be reviewed by a colleague who is proficient in English grammar in the medical context. Most of the medical writings received in biomedical journals require substantial modifications, and it is unlikely that most writings will be accepted in its original form. Keep in mind that your manuscript will be read by at least two reviewers who will be your most staunch critics. They will point out your mistakes, ambiguous areas, as well as clarifications before your manuscript has an adequate quality for publication. When the article has adequate potential to be published, these reviewers will send you constructive comments that will facilitate the changes to your original manuscript. Changes may be minimal or require substantial modifications. Do not pass out! Make the modifications suggested by your reviewers as they are the experts and will take you by the hand until your writing has an optimal structure for final publication. ${ }^{7}$ If your writing is dismal, it will be rejected immediately and you must redo it almost completely. If this is your case, don't give up and pay more attention to your protocol, rewrite your ideas and results with special attention to the discussion and conclusions. Write each sentence carefully. Only include the necessary tables and figures. It is a mistake to think that using many tables is better. Edit your tables according to the guidelines of the journal that you are going to send your manuscript and keep in mind that neither the editor nor the reviewers will condense your tables. Pay special attention to this topic or you will find that your manuscript can be rejected.

In relation to your references, use only the citations you consulted and that you consider are useful to support your writing or so that the readers can use to expand their information. Write it correctly and don't settle for copy-paste without reading them carefully. It is common for citations to be misspelled. These are just some recommendations that are useful to know before and during the preparation of your manuscript. ${ }^{2}$

a) You should avoid a confusing and jargon-filled writing that is difficult to read, even for medical researchers. Use simple medical language.

b) Vague, ambiguous or invented publications induce medical errors that harm our patients.

c) Bad communication limits the impact of medical research, so doctors and patients finally suffer too. 
d) Look for patterns of excellent medical writing and learn by imitation.

e) A good guiding principle: strive to be clear, concise and simple enough not to confuse an intelligent non-expert.

f) Keep in mind that research requires incredible amounts of time, effort and money.

g) Authors who put little effort into presenting their work are clearly damaging their profession, to the public and themselves.

\section{Peer review}

Keep in mind that peer review is essential in the editorial process of a biomedical journal. This process has very heterogeneous variants according to each journal, editors and reviewers. The uniformity of peer review should be essential since manuscripts should generally be attached to international guidelines for research studies and in particular to the specific recommendations of each journal. Frank ${ }^{8}$ found that $96 \%$ of journals asked reviewers to accept or reject manuscripts, $72 \%$ requested the reviewers to evaluate the novelty of the manuscripts, $69 \%$ demanded clarity assessments and only $51 \%$ required evaluations of the conclusions. Only $46 \%$ of journals reminded reviewers that manuscripts were confidential documents, $51 \%$ provided a separate cover letter and $25 \%$ provided extensive review instructions. The study by Kravitz et al. ${ }^{9}$ found that $28 \%$ of reviewers recommended rejecting the manuscripts, while the general rate of rejection among the editors was $48 \%$; $88 \%$ when all reviewers of a manuscript agreed to rejection (7\% of manuscripts), and $20 \%$ when all reviewers agreed that the manuscript should not be rejected ( $48 \%$ of manuscripts) $(\mathrm{p}<0.01)$. The rejection rate can be as high as $93 \%$ with expert reviewers. ${ }^{10}$

Although mentioning whether or not there is a conflict of interest in your manuscript does not have an effect on the opinion of the reviewers, it is always advisable to mention it, ${ }^{11}$ especially when it comes to pharmacological investigations that are related to the pharmacological industry. There is a positive trend in the peer review process to support researchers to improve their manuscripts and reduce the delay in editorial processing and eventual publication. The way to go is still long since the goal is to improve the academic quality of the publications. $7,11,12,13$

Fortunately, not all medical professionals are trained or interested in publishing their experiences. If you are a reader with the desire to publish read about it, study how to plan a research protocol, familiarize yourself with the medical writing beyond what you did with the thesis that you wrote when you graduated from medical school and when you finished your residence or fellowship. I strongly recommend a small book entitled Becoming an academic writer written by Patricia Goodson, that I frequently use..$^{14}$ It is a very useful practical guide, even for experienced writers, reviewers and editors. Regardless of your professional age, it is always a good time to publish your experiences. I assure you that there will always be colleagues interested in reading and using them in daily practice with their patients.
Science evolves by leaps and bounds and it is now impossible to be updated in the profession we have chosen as a way of life. There are so many publications that it is very difficult to choose which ones we must read, which are useful for our medical practice. Each author considers that his/her writing is good, that his/her experience can be useful to other colleagues and that editors and reviewers should publish and disseminate. Contributing to medical science is a pleasure that requires honesty, ethics, and seriousness in every investigation that eventually benefits patients.

It is beyond the scope of this editorial to give easy recipes on how to write well. Learn medical writing and try to be clear, concise and simple in your manuscripts.

\section{References}

1. Ben Saad H. Scientific medical writing in practice: How to succeed the writing style? Tunis Med. 2019;97(2):273-285.

2. Collier R. A call for clarity and quality in medical writing. CMAJ. 2017;189(46):E1407.

3. Whizar LV, Chávez PE, Casillas MR. Analgesia peridural con morfina en dolor por cáncer terminal. Rev Clin Esp. 1982;166:299-301.

4. Whizar-Lugo VM. Un siglo de analgesia pre-emptiva y preventiva La historia de George Washington Crile (1864-1943). Actas Peru Anestesiol. 2017;22(1):31-40.

5. Ben Saad H. Scientific medical writing in practice: the «IMR@D®» format. Tunis Med. 2019;97(3):407-425.

6. Maranhão-Filho P. Suggestions for authors of medical articles. Arq Neuropsiquiatr. 2017;75(2):114-116.

7. Grant MJ. Peer review at the health information and libraries journal. Health Info Libr J. 2014;31(4):251-253.

8. Frank E. Editors' requests of peer reviewers: a study and a proposal. Prev Med. 1996;25(2):102-104.

9. Kravitz RL, Franks P, Feldman MD, et al. Editorial peer reviewers' recommendations at a general medical journal: Are they reliable and do editors care? PLoS One. 2010;5(4):e10072.

10. Vintzileos AM, Ananth CV, Odibo AO, et al. The relationship between a reviewer's recommendation and editorial decision of manuscripts submitted for publication in obstetrics. Am J Obstet Gynecol. 2014;211(6):703.e1-5.

11. John LK, Loewenstein G, Marder A, et al. Effect of revealing authors' conflicts of interests in peer review: randomized controlled trial. $B M J$ 2019;367:15896.

12. Sposato LA, Ovbiagele B, Johnston SC, et al. A peek behind the curtain: peer review and editorial decision making at stroke. Ann Neurol. 2014;76(2):151-158.

13. Audisio RA, Stahel RA, Aapro MS, et al. Successful publishing: how to get your paper accepted. Surg Oncol. 2009;18(4):350-356.

14. Goodson P. Becoming an academic writer. SAGE Publication, Inc. Los Angeles USA. 2017. 